\title{
Many-body theory of excitation dynamics in an ultracold Rydberg gas
}

\author{
C. Ates, ${ }^{1}$ T. Pohl,${ }^{2}$ T. Pattard ${ }^{*}{ }^{1}$ and J.M. Rost ${ }^{1}$ \\ ${ }^{1}$ Max Planck Institute for the Physics of Complex Systems, \\ Nöthnitzer Straße 38, D-01187 Dresden, Germany \\ ${ }^{2}$ ITAMP, Harvard-Smithsonian Center for Astrophysics, \\ 60 Garden Street, MS14, Cambridge, MA 02138, USA
}

(Dated: October 28, 2018)

\begin{abstract}
We develop a theoretical approach for the dynamics of Rydberg excitations in ultracold gases, with a realistically large number of atoms. We rely on the reduction of the single-atom Bloch equations to rate equations, which is possible under various experimentally relevant conditions. Here, we explicitly refer to a two-step excitation-scheme. We discuss the conditions under which our approach is valid by comparing the results with the solution of the exact quantum master equation for two interacting atoms. Concerning the emergence of an excitation blockade in a Rydberg gas, our results are in qualitative agreement with experiment. Possible sources of quantitative discrepancy are carefully examined. Based on the two-step excitation scheme, we predict the occurrence of an antiblockade effect and propose possible ways to detect this excitation enhancement experimentally in an optical lattice as well as in the gas phase.

PACS numbers: 32.70.Jz,32.80.Rm,34.20.Cf
\end{abstract}

\section{INTRODUCTION}

The possibility to routinely create samples of ultracold gases in the $\mu$-Kelvin regime has opened a new avenue to the investigation of interacting many-particle systems. At such temperatures, the thermal velocities of the atoms are low enough that the atoms move a negligible distance over the duration of the experiment. Hence, (thermal) collisions are not relevant and it is possible to study quasi-static interactions between the particles.

For densities of a dilute ultracold but non-degenerate gas typical for atoms in magneto-optical traps, the interaction between ground state atoms is very weak. Rydberg atoms, on the other hand, can strongly interact among each other, even in a dilute gas, due to their large polarizability which scales with the principal quantum number $n$ as $n^{7}$. This scaling allows an accurate control over their interactions [1] over a huge range by varying $n$. In contrast to amorphous solids, with which ultracold Rydberg gases share some similarities, the atoms are practically stationary on the timescale of electronic dynamics because of their low thermal kinetic energy [2, 3].

A striking consequence of the strong Rydberg-Rydberg interaction is the so-called "dipole blockade", i.e., a suppression of Rydberg excitations due to an induced dipole coupling of the Rydberg atoms to their environment. This phenomenon was first considered theoretically in proposals to build fast quantum logic gates [4], to improve the resolution of atomic clocks [5] and to create single-atom and single-photon sources [6]. It was experimentally verified for second-order dipole-dipole (or van der Waals) coupling between the Rydberg atoms $[7,8]$ by

\footnotetext{
*present address: APS Editorial Office, 1 Research Road, Ridge,
} NY 11961, USA measuring the density of the Rydberg atoms as a function of increasing laser intensity, atomic density or principal quantum number, i.e., as a function of increasing interaction strength. By applying and varying an external electric field the blockade effect was also demonstrated for a direct (i.e. first order) dipole-dipole interaction of the Rydberg atoms and it was shown that the suppression of excitations is particularly pronounced at the so called Förster resonances [9]. Furthermore, it was shown that the blockade effect also leads to a quenching of the probability distribution for the excitation to the Rydberg state [10, 11, 12].

The theoretical description of this laser-driven, interacting many-particle system is challenging. In [7] a mean field approach was used and the Bloch equations for a single Rydberg atom in a sphere were solved. Within the sphere, embedded in a constant background density of Rydberg atoms, no further excitations were allowed. With the help of a fit parameter the experimental results of [7] could be reproduced.

The system was also investigated by solving the manyparticle Schrödinger equation numerically [13]. There, intelligent use was made of the fact that the blockade itself reduces the number of atoms which can be excited which allows a substantial reduction in the number of states that had to be considered for the calculations. Yet, the number of atoms that could be simulated was still so small that appropriate boundary conditions had to be used to establish contact with the experiments. However, experiments using a two-step (three-level) excitation scheme could not be described since important effects, such as radiative decay, were not included.

Here, we focus in particular on the two-step excitation scheme, used in the experiments [8, 10], where the intermediate level decays radiatively. As we will show, this leads to a reduction of the description of the Rydberg excitation dynamics in a single atom to a rate equation 
which in turn enables us to formulate a quasi-classical approach taking fully into account all atoms in the excitation volume and all interactions of the Rydberg atoms.

Experimentally, a gas of atoms is prepared in a magneto-optical trap (MOT) with peak densities up to $10^{11} \mathrm{~cm}^{-3}$ at temperatures of about $100 \mu \mathrm{K}$. Under these conditions the gas is far from the quantum degenerate regime and can be viewed as a classical ideal gas. Furthermore, the laser intensities used in [8] and [10] are relatively low, so that coherent coupling of the atoms by the laser field, e.g., through stimulated emission and reabsorption of photons, is negligible. However, the interaction of the individual atoms with the laser fields has to be treated quantum mechanically.

Our approach is based on the observation that, under the conditions of the experiments [8] and [10], the description of the single-atom excitation dynamics can be reduced substantially to a single rate equation using an adiabatic approximation for the coherences. Despite the approximations made, the rate equation accurately describes the population dynamics of the Rydberg state, including non-trivial effects like the Autler-Townes splitting of the excitation line. This simplification in the description of the single-atom dynamics is the key that ultimately allows us to fully account for the correlated many-particle dynamics with a simple Monte-Carlo sampling, thereby reducing greatly the complexity of a full quantum treatment.

The paper is organized as follows. In Sec. Пwe present the approach, which enables us to describe the dynamics in an ultracold gas of interacting three-level atoms using a many-body rate equation. Starting from the full quantum master equation, we justify our approximations first on the single-atom level (Sec. (IIA), then for the interacting system (Sec. IIB) and finally describe how the Rydberg-Rydberg interaction is included in our description (Sec. IIC). For two interacting atoms, we compare the results of our rate equation with the solution of the quantum master equation (Sec. III). In Sec. IV] we compare the results of the simulations for a realistic number of atoms with experimental data and comment on the possibility to experimentally observe an interaction induced enhancement of Rydberg excitation ("antiblockade"). Section $\nabla$ summarizes the results.

Throughout the paper atomic units will be used unless stated otherwise.

\section{TWO-STEP RYDBERG EXCITATION IN AN ULTRACOLD GAS}

\section{A. Dynamics of the non-interacting system}

In what follows, we will discuss a two-step cwexcitation scheme for the Rydberg state (see Fig. 1), as typically used in experiments. In the first step, the atom is excited from its ground state $|g\rangle$ to an intermediate level $|m\rangle$ with a transition strength given by the Rabi

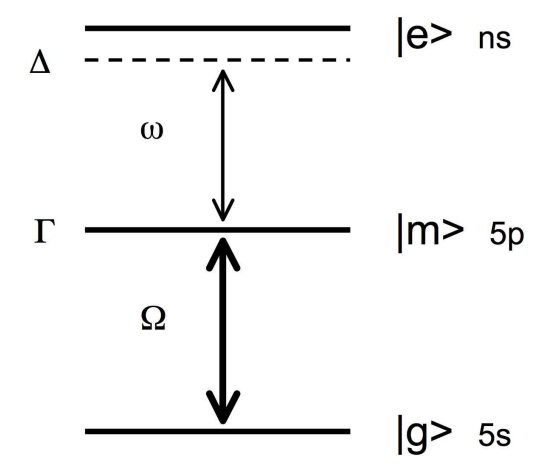

FIG. 1: Sketch of the two-step excitation scheme for rubidium.

frequency $\Omega$. The photon for this step is typically provided by the MOT lasers, which are tuned on resonance with the transition $|g\rangle \rightarrow|m\rangle$ during the time of Rydberg excitation.

In the second step, a separate tunable laser drives the transition between the intermediate level and the desired Rydberg state $|e\rangle$ with Rabi frequency $\omega$, where in this step we allow for a detuning $\Delta$ from resonant excitation. The decay of the intermediate level with rate $\Gamma$ has to be taken into account, as its radiative lifetime is typically shorter than the pulse duration. On the other hand, the lifetime of the Rydberg state is much longer so that its decay can be neglected.

The coherent dynamics of $N$ non-interacting threelevel atoms coupled to the two laser fields is governed in the interaction picture by the Hamiltonian $H_{0}$,

$$
H_{0}=H_{\Delta}+H_{A L} \equiv \sum_{i}^{N} h_{\Delta}^{(i)}+\sum_{i}^{N} h_{A L}^{(i)},
$$

where

$$
\begin{aligned}
h_{\Delta}^{(i)}= & \Delta\left|e_{i}\right\rangle\left\langle e_{i}\right|, \\
h_{A L}^{(i)}= & \frac{\Omega}{2}\left(\left|m_{i}\right\rangle\left\langle g_{i}|+| g_{i}\right\rangle\left\langle m_{i}\right|\right) \\
& +\frac{\omega}{2}\left(\left|e_{i}\right\rangle\left\langle m_{i}|+| m_{i}\right\rangle\left\langle e_{i}\right|\right)
\end{aligned}
$$

describe the interaction of the levels of atom $i$ with the laser beams.

The time evolution of the system including the decay of the intermediate level is then given by a quantum master equation for the $N$-particle density matrix $\hat{\rho}^{(N)} \equiv \hat{\boldsymbol{\rho}}$,

$$
\frac{\mathrm{d}}{\mathrm{d} t} \hat{\boldsymbol{\rho}}=-i\left[H_{0}, \hat{\boldsymbol{\rho}}\right]+\mathcal{L}[\hat{\boldsymbol{\rho}}]
$$

where the spontaneous decay of level $|m\rangle$ is included via the Lindblad operator $\mathcal{L}$. In general, the rate of spontaneous decay of an atom is influenced by the presence of other atoms through a coupling mediated by the radiation field, which can account for collective effects like superradiance. The strength of this coupling is determined by the dimensionless quantity $x_{i j} \equiv 2 \pi\left|\mathbf{r}_{i}-\mathbf{r}_{\mathbf{j}}\right| / \lambda$, 
which measures the atom-atom distance in units of the wavelength $\lambda$ of the $|g\rangle \rightarrow|m\rangle$ transition. For $x_{i j} \ll 1$ the spontaneous decay of an atom is strongly affected by its neighbors, while for $x_{i j} \gg 1$ the atoms radiate independently. In typical experiments with ultracold gases, the mean atomic distance between atoms is $a \sim 5 \mu \mathrm{m}$. For the $5 s \rightarrow 5 p$ transition of $\mathrm{Rb}$ this corresponds to $x_{i j} \sim 40$. Hence, the collective decay is negligible and the Lindblad operator can be written as a sum of singleatom operators,

$$
\mathcal{L}=\Gamma \sum_{i}^{N}\left(L_{i} \hat{\boldsymbol{\rho}} L_{i}^{\dagger}-\frac{1}{2} L_{i}^{\dagger} L_{i} \hat{\boldsymbol{\rho}}-\frac{1}{2} \hat{\boldsymbol{\rho}} L_{i}^{\dagger} L_{i}\right)
$$

with

$$
L_{i}=\left|g_{i}\right\rangle\left\langle m_{i}\right| \quad \text { and } \quad L_{i}^{\dagger}=\left|m_{i}\right\rangle\left\langle g_{i}\right| .
$$

Hence, the dynamics of the atoms is completely decoupled and the $N$-atom density matrix factorizes, $\hat{\boldsymbol{\rho}}=$ $\hat{\rho}_{1}^{(1)} \otimes \ldots \otimes \hat{\rho}_{N}^{(1)}$. The time evolution of a non-interacting gas of three-level atoms is therefore completely determined by the master equation for the single-atom density matrix $\hat{\rho}_{k}^{(1)} \equiv \hat{\rho}$, i.e., the optical Bloch equations (OBE) for a three-level atom,

$$
\begin{aligned}
\dot{\rho}_{\mathrm{gg}}= & i \frac{\Omega}{2}\left(\rho_{\mathrm{gm}}-\rho_{\mathrm{mg}}\right)+\Gamma \rho_{\mathrm{mm}} \\
\dot{\rho}_{\mathrm{mm}}= & -i \frac{\Omega}{2}\left(\rho_{\mathrm{gm}}-\rho_{\mathrm{mg}}\right) \\
& +i \frac{\omega}{2}\left(\rho_{\mathrm{me}}-\rho_{\mathrm{em}}\right)-\Gamma \rho_{\mathrm{mm}} \\
\dot{\rho}_{\mathrm{ee}}= & -i \frac{\omega}{2}\left(\rho_{\mathrm{me}}-\rho_{\mathrm{em}}\right) \\
\dot{\rho}_{\mathrm{gm}}= & -i \frac{\Omega}{2}\left(\rho_{\mathrm{mm}}-\rho_{\mathrm{gg}}\right)+i \frac{\omega}{2} \rho_{\mathrm{ge}}-\frac{\Gamma}{2} \rho_{\mathrm{gm}} \\
\dot{\rho}_{\mathrm{me}}= & -i \Delta \rho_{\mathrm{me}}-i \frac{\omega}{2}\left(\rho_{\mathrm{ee}}-\rho_{\mathrm{mm}}\right) \\
& -i \frac{\Omega}{2} \rho_{\mathrm{ge}}-\frac{\Gamma}{2} \rho_{\mathrm{me}} \\
\dot{\rho}_{\mathrm{ge}}= & -i \Delta \rho_{\mathrm{ge}}-i \frac{\Omega}{2} \rho_{\mathrm{me}}+i \frac{\omega}{2} \rho_{\mathrm{gm}} \\
\rho_{\beta \alpha}= & \left(\rho_{\alpha \beta}\right)^{\star} \quad \text { for } \quad \alpha \neq \beta .
\end{aligned}
$$

As usual, the level populations are described by the diagonal elements of the density matrix, whereas the offdiagonal elements, i.e., the coherences, contain the information about the transition amplitudes between the levels. Conservation of probability leads to the sum rule

$$
\sum_{\alpha} \rho_{\alpha \alpha}=1
$$

for the populations so that 8 independent variables remain to be solved for.

This single-atom description is too complex to serve as the basis of a tractable description for the many-particle system. Fortunately, under the set of relevant experimental parameters Eq.(6) simplifies substantially. In the experiments [8, 10], the upper transition is much more weakly driven than the lower one $(\omega \ll \Omega)$ due to the different transition dipole moment of the respective excitation. This defines two well separated time scales, such that the Rydberg transition $|m\rangle \rightarrow|e\rangle$ is slow compared to the pump transition $|g\rangle \rightarrow|m\rangle$. Thus, the time evolution of the system is governed by the slow Rydberg transition in the sense that the coherences of the fast pump transition will adiabatically follow the slow dynamics of the Rydberg transition.

Furthermore, the decay rate of the intermediate level is much larger than the Rabi frequency of the upper transition $(\Gamma \gg \omega)$ implying that the populations will evolve only slightly over a time $\Gamma^{-1}$. Hence, dephasing of the atomic transition dipole moments, i.e., damping of the oscillations of coherences, is fast compared to the dynamics of the Rydberg population.

Under these conditions, the coherences can be expressed as a function of the populations at each instant of time, i.e., their dynamics can be eliminated adiabatically [14] by setting

$$
\dot{\rho}_{\alpha \beta}=0 \quad \text { for } \quad \alpha \neq \beta .
$$

Solving the algebraic equations arising from (6) and (8) for the populations, making use of (77) and inserting into the differential equation for $\rho_{\mathrm{mm}}$ and $\rho_{\mathrm{ee}}$ one arrives at

$$
\begin{aligned}
\dot{\rho}_{\mathrm{mm}} & =q_{1} \rho_{\mathrm{mm}}+q_{2} \rho_{\mathrm{ee}}+q_{3} \\
\dot{\rho}_{\mathrm{ee}} & =q_{4} \rho_{\mathrm{mm}}+q_{5} \rho_{\mathrm{ee}}+q_{6},
\end{aligned}
$$

where the coefficients $q_{k}=q_{k}(\Omega, \omega, \Gamma, \Delta)$ are some functions of the parameters describing the excitation dynamics of the three-level system.

To simplify further, we note that within the adiabatic approximation (8) the dynamics of the population difference $\rho_{\mathrm{mm}}-\rho_{\mathrm{gg}}$ can be neglected for times $t>1 / 2 \Gamma$. This can be verified by direct integration of $\dot{\rho}_{\mathrm{mm}}-\dot{\rho}_{\mathrm{gg}}$ from the OBE, which shows that the dynamics of the population difference is proportional to $1-\exp (-2 \Gamma t)$ and thus reaches its saturation limit at a timescale on the order of $1 / 2 \Gamma$. Using the sum rule (7) this leads to the relation

$$
2 \dot{\rho}_{\mathrm{mm}}+\dot{\rho}_{\mathrm{ee}}=0,
$$

which can be used to eliminate the population of the intermediate level occurring in (9). Finally, one arrives at a single differential equation for $\rho_{\mathrm{ee}}$

$$
\dot{\rho}_{\mathrm{ee}}=-\frac{\gamma_{\uparrow}}{\rho_{\mathrm{ee}}^{\infty}} \rho_{\mathrm{ee}}+\gamma_{\uparrow},
$$

which can readily be solved to give

$$
\rho_{\mathrm{ee}}(t)=\rho_{\mathrm{ee}}^{\infty}\left(1-\exp \left[-\frac{\gamma_{\uparrow}}{\rho_{\mathrm{ee}}^{\infty}} t\right]\right),
$$

where $\rho_{\mathrm{ee}}^{\infty}=\rho_{\mathrm{ee}}^{\infty}(\Omega, \omega, \Gamma, \Delta)$ denotes the steady-state occupation of level $|e\rangle$ and $\gamma_{\uparrow}=\gamma_{\uparrow}(\Omega, \omega, \Gamma, \Delta)$ is the rate 

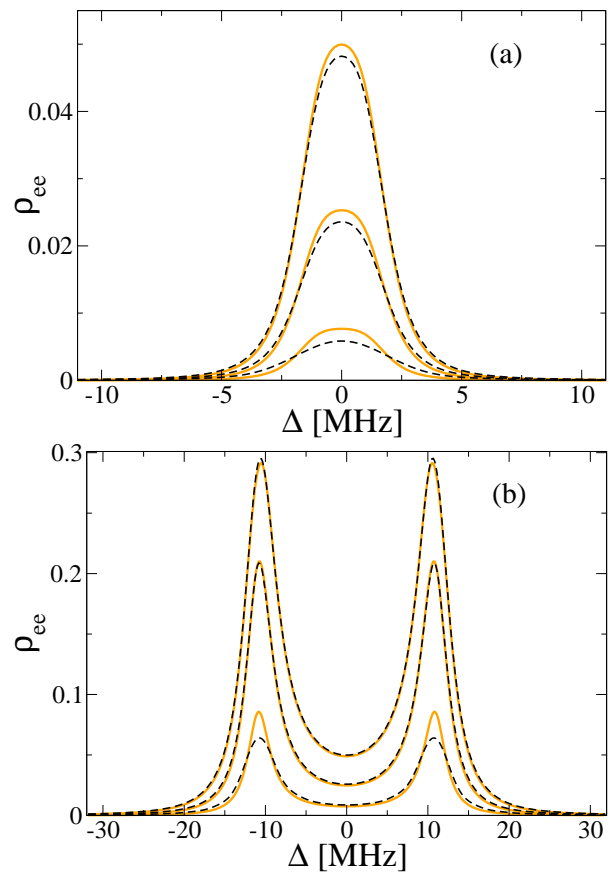

FIG. 2: Population of the Rydberg level for the three-level system of Fig. 1 according to the RE Eq.(14) (solid lines) and OBE Eq. (6) (dashed lines) for different pulse lengths: $0.3 \mu \mathrm{s}$ (lowest pair of curves), $1.0 \mu \mathrm{s}$ (middle pair) and $2.0 \mu \mathrm{s}$. The parameters in $\mathrm{MHz}$ are: (a) $(\Omega, \omega, \Gamma)=(4,0.2,6)$ in (a) and $(22.1,0.8,6)$ in $(b)$.

for populating the Rydberg level for short times. The expressions for $\gamma_{\uparrow}$ and $\rho_{\mathrm{ee}}^{\infty}$ are given in the Appendix. Here we note only that in the limit $\Omega \gg \Gamma \gg \omega$ they reduce to

$$
\gamma_{\uparrow}=\frac{\Gamma \omega^{2} / \Omega^{2}}{2\left(1-4 \Delta^{2} / \Omega^{2}\right)^{2}} \quad, \quad \rho_{\mathrm{ee}}^{\infty}=\frac{1}{1+8 \Delta^{2} / \Omega^{2}},
$$

which shows that the resonant excitation rate is proportional to $(\omega / \Omega)^{2}$.

Introducing an effective ground state $\rho_{\mathrm{gg}}^{\mathrm{eff}}=1-\rho_{\mathrm{ee}}$, one can write (11) in the form of a rate equation (RE) for an effective two-level atom

$$
\dot{\rho}_{\mathrm{ee}}(t)=\gamma_{\uparrow} \rho_{\mathrm{gg}}^{\mathrm{eff}}-\gamma_{\downarrow} \rho_{\mathrm{ee}},
$$

with de-excitation rate

$$
\gamma_{\downarrow}=\gamma_{\uparrow}\left(\frac{1-\rho_{\mathrm{ee}}^{\infty}}{\rho_{\mathrm{ee}}^{\infty}}\right) .
$$

A comparison of the solutions of the OBE (6) and the RE (14) for the Rydberg populations as function of the detuning $\Delta$ is shown in Fig. 2 for different pulse lengths. The parameters correspond to those of the experiments [10] and [8]. The agreement of the solutions is generally good and becomes even better for longer pulses. For the parameters of experiment [10] the convergence of the $\mathrm{RE}$ solution to that of the OBE in the region around $\Delta=0$ is slower as a function of pulse length. This is due to $\Omega<\Gamma$ which indicates that it is not fully justified to neglect the nonlinear short-time population dynamics.

The RE reproduces the Autler-Townes splitting of the intermediate level $|m\rangle$ manifest in a splitting of the Rydberg line, proportional to $\Omega$ for short times. The splitting is transient, as the steady state with its single central peak is approached for long times when the Rydberg population reaches the saturation limit. A detailed analysis of the peak structure of the Rydberg populations in this system, especially the occurrence of the Autler-Townes splitting and its impact on the excitation blockade, has been given in [15].

For future reference, we will cast the single-atom RE (14) into a form which will be used for the simulation of the interacting many-particle system. To this end, we denote the state of the atom by $\sigma$, where $\sigma=1$ if the atom is in the Rydberg state, and $\sigma=0$ otherwise. Furthermore, we define the rate of change for the state $\sigma$,

$$
\gamma(\Delta, \sigma) \equiv(1-\sigma) \gamma_{\uparrow}(\Delta)+\sigma \gamma_{\downarrow}(\Delta),
$$

which describes excitation of the atom if it is in the (effective) ground state $(\sigma=0)$ and de-excitation if it is in the excited state $(\sigma=1)$. Using these definitions, we can combine (14) which determines $\rho_{\mathrm{ee}}(t)$ and the corresponding equation for $\rho_{\mathrm{gg}}^{\mathrm{eff}}(t)$ in the form of an evolution equation for the single-atom state distribution function $p(\sigma)$,

$$
\frac{d p(\sigma)}{d t}=\sum_{\sigma^{\prime}} T\left(\sigma, \sigma^{\prime}\right) p\left(\sigma^{\prime}\right)
$$

with $p(0)=1-\rho_{\mathrm{ee}}, p(1)=\rho_{\mathrm{ee}}$ and the transition rate matrix

$$
T\left(\sigma, \sigma^{\prime}\right)=-\gamma(\Delta, \sigma) \delta_{\sigma, \sigma^{\prime}}+\gamma(\Delta, 1-\sigma) \delta_{1-\sigma, \sigma^{\prime}} .
$$

The first term of (18) describes the transition $\sigma \rightarrow 1-\sigma$, through which the system can leave the state $\sigma$, while the opposite process $(1-\sigma \rightarrow \sigma)$, which brings the system into the state $\sigma$, is described by the second term.

Proceeding to the case of $N$ non-interacting atoms, we define the many-particle state $\boldsymbol{\sigma}$ as the configuration containing all single-atom states $\sigma_{i}$, i.e., $\boldsymbol{\sigma} \equiv$ $\left\{\sigma_{1}, \ldots, \sigma_{i}, \ldots \sigma_{N}\right\}$ and $\boldsymbol{\sigma}_{i}$ as the many-body configuration which is identical to $\sigma$ except for the state of atom $i$, i.e., $\boldsymbol{\sigma}_{i} \equiv\left\{\sigma_{1}, \ldots, 1-\sigma_{i}, \ldots, \sigma_{N}\right\}$. If we finally use the notation $\gamma(\Delta, \boldsymbol{\sigma}) \equiv \sum_{i} \gamma\left(\Delta, \sigma_{i}\right)$ and $\delta_{\boldsymbol{\sigma}, \boldsymbol{\sigma}^{\prime}} \equiv$ $\delta_{\sigma_{1}, \sigma_{1}^{\prime}} \cdots \delta_{\sigma_{N}, \sigma_{N}^{\prime}}$, the matrix of the transition rates generalizes to

$$
T\left(\boldsymbol{\sigma}, \boldsymbol{\sigma}^{\prime}\right)=-\gamma(\Delta, \boldsymbol{\sigma}) \delta_{\boldsymbol{\sigma}, \boldsymbol{\sigma}^{\prime}}+\sum_{i} \gamma\left(\Delta, 1-\sigma_{i}\right) \delta_{\boldsymbol{\sigma}_{i}, \boldsymbol{\sigma}^{\prime}},
$$

and the evolution equation for the many-body state distribution function $P(\boldsymbol{\sigma})$ can be written in a closed form as

$$
\frac{d P(\boldsymbol{\sigma})}{d t}=\sum_{\boldsymbol{\sigma}^{\prime}} T\left(\boldsymbol{\sigma}, \boldsymbol{\sigma}^{\prime}\right) P\left(\boldsymbol{\sigma}^{\prime}\right)
$$


For non-interacting particles the rate $\gamma$ depends (besides on the laser detuning) only on the state of particle $i$, i.e., on $\sigma_{i}$. However, this is no longer true in the interacting case and $\gamma$ will depend on the entire many-body configuration.

\section{B. Correlated many-particle dynamics}

In order to study the correlated dynamics of the interacting many-particle system, we have to add the Hamiltonian describing the Rydberg-Rydberg interaction

$$
H_{R R}=\frac{1}{2} \sum_{i, j(i \neq j)} U_{i j}\left|e_{i}, e_{j}\right\rangle\left\langle e_{i}, e_{j}\right|
$$

to $H_{0}$ (c.f. eq. (1)), where $U_{i j}$ is the interaction energy of a pair of Rydberg atoms at a distance $r_{i j} \equiv\left|\mathbf{r}_{i}-\mathbf{r}_{j}\right|$. The quantum master equation (3) then reads

$$
\frac{\mathrm{d}}{\mathrm{d} t} \hat{\boldsymbol{\rho}}=-i\left[H_{0}+H_{R R}, \hat{\boldsymbol{\rho}}\right]+\mathcal{L}[\hat{\boldsymbol{\rho}}]
$$

with the Lindblad operator given in (4).

To see which terms of the master equation are affected by the inclusion of the Rydberg-Rydberg interaction we consider the commutator $\left[H_{\Delta}+H_{R R}, \boldsymbol{\rho}\right]$ in the manybody basis $|\boldsymbol{\alpha}\rangle \equiv\left|\alpha_{1}, \ldots \alpha_{N}\right\rangle=\left|\alpha_{1}\right\rangle \cdots\left|\alpha_{N}\right\rangle$, where $\left|\alpha_{i}\right\rangle$ denotes the state of atom $i$,

$$
\left(\left[H_{\Delta}+H_{R R}, \boldsymbol{\rho}\right]\right)_{\boldsymbol{\alpha} \boldsymbol{\beta}}=\sum_{i}\left[\left(\Delta+\sum_{j(i \neq j)} \frac{U_{i j}}{2} \delta_{\alpha_{j}, e}\right) \delta_{\alpha_{i}, e}-\left(\Delta+\sum_{j(i \neq j)} \frac{U_{i j}}{2} \delta_{\beta_{j}, e}\right) \delta_{\beta_{i}, e}\right] \boldsymbol{\rho}_{\boldsymbol{\alpha} \boldsymbol{\beta}},
$$

and rewrite it (using the conservation of probabilities for each atom, i.e., $1=\delta_{\mu_{k}, g}+\delta_{\mu_{k}, m}+\delta_{\mu_{k}, e} \equiv \delta_{\mu_{k}, \tilde{g}}+\delta_{\mu_{k}, e}$, and the symmetry of the Rydberg-Rydberg interaction, $\left.U_{i j}=U_{j i}\right)$ as

$$
\begin{aligned}
\left(\left[H_{\Delta}+H_{R R}, \boldsymbol{\rho}\right]\right)_{\boldsymbol{\alpha} \boldsymbol{\beta}}= & \sum_{i}\left(\delta_{\alpha_{i}, e} \delta_{\beta_{i}, \tilde{g}}-\delta_{\alpha_{i}, \tilde{g}} \delta_{\beta_{i}, e}\right)\left[\Delta+\sum_{j \neq i} U_{i j} \delta_{\alpha_{j}, e} \delta_{\beta_{j}, e}\right] \boldsymbol{\rho}_{\boldsymbol{\alpha} \boldsymbol{\beta}} \\
& +\sum_{i, j(i \neq j)} \frac{U_{i j}}{2}\left(\delta_{\alpha_{i}, e} \delta_{\alpha_{j}, e} \delta_{\beta_{i}, \tilde{g}} \delta_{\beta_{j}, \tilde{g}}-\delta_{\alpha_{i}, \tilde{g}} \delta_{\alpha_{j}, \tilde{g}} \delta_{\beta_{i}, e} \delta_{\beta_{j}, e}\right) \boldsymbol{\rho}_{\boldsymbol{\alpha} \boldsymbol{\beta}}
\end{aligned}
$$

In the first term of (24) the Rydberg-Rydberg interaction shows up as an additional (local) detuning of an atom at $\mathbf{r}_{i}$, whenever the atom at $\mathbf{r}_{j}$ is in the Rydberg state (i.e., if $\alpha_{j}=\beta_{j}=e$ ). In particular, no additional coherences are generated by the Rydberg-Rydberg interaction and, therefore, this term does not change the structure of the master equation as compared to the noninteracting case.

The second term describes direct transitions between states where atoms $i$ and $j$ are not in the Rydberg state and the state where the atoms form a Rydberg pair. These transitions require the simultaneous absorption or emission of at least two photons and are thus higher order processes. The dynamics of these multi-photon processes is very slow compared to all other transitions in the system, therefore it can be neglected (see also the discussion in sections III and IVA), i.e., the commutator (24) can be approximated as

$$
\left(\left[H_{\Delta}+H_{R R}, \boldsymbol{\rho}\right]\right)_{\boldsymbol{\alpha} \boldsymbol{\beta}} \approx \sum_{i}\left(\delta_{\alpha_{i}, e} \delta_{\beta_{i}, \tilde{g}}-\delta_{\alpha_{i}, \tilde{g}} \delta_{\beta_{i}, e}\right)\left[\Delta+\sum_{j \neq i} U_{i j} \delta_{\alpha_{j}, e} \delta_{\beta_{j}, e}\right] \boldsymbol{\rho}_{\boldsymbol{\alpha} \boldsymbol{\beta}} .
$$

Thus, within this approximation, we recover the simple

picture which is commonly used for the explanation of 
the dipole blockade effect, namely that a highly excited atom shifts the Rydberg levels of nearby atoms out of resonance with the excitation laser.

By neglecting multi-photon transitions, the structure of the master equation is not changed compared to the non-interacting system and we can perform the adiabatic approximation discussed above. Identifying finally $\delta_{\alpha_{j}, e} \delta_{\beta_{j}, e}$ with $\sigma_{j}$, it is straightforward to generalize Eq. (19) to the interacting case,

$$
T\left(\boldsymbol{\sigma}, \boldsymbol{\sigma}^{\prime}\right)=-\gamma(\Delta, \boldsymbol{\sigma}) \delta_{\boldsymbol{\sigma}, \boldsymbol{\sigma}^{\prime}}+\sum_{i} \gamma\left(\Delta_{i}, 1-\sigma_{i}\right) \delta_{\boldsymbol{\sigma}_{i}, \boldsymbol{\sigma}^{\prime}},
$$

where now $\gamma(\Delta, \boldsymbol{\sigma})=\sum_{i} \gamma\left(\Delta, \delta_{i}, \sigma_{i}\right)$ and all atoms are coupled by the energetic shift caused by the RydbergRydberg interaction

$$
\Delta_{i}=\Delta+\delta_{i} \equiv \Delta+\sum_{j \neq i} \sigma_{j} U_{i j},
$$

so that in the interacting case the rate for a state change $\gamma\left(\Delta, \delta_{i}, \sigma_{i}\right)$ for the atom $i$ depends on the entire manybody configuration through the local detuning $\delta_{i}$.

The above approximations simplify the description of the correlated many-particle dynamics to a high degree, since a particular many-particle configuration $\sigma$ is directly coupled to "only" $N$ configurations $\boldsymbol{\sigma}^{\prime}$ by the transition rate matrix $T\left(\boldsymbol{\sigma}, \boldsymbol{\sigma}^{\prime}\right)$, which has to be compared to the available number of $2^{N}$ many-particle states. To explicitly show this simplification, we insert (26) into the evolution equation (20) of the state distribution function, perform the sum over $\boldsymbol{\sigma}^{\prime}$ and finally arrive at

$\frac{d P(\boldsymbol{\sigma})}{d t}=-\sum_{i}^{N} \gamma\left(\Delta, \delta_{i}, \sigma_{i}\right) P(\boldsymbol{\sigma})+\sum_{i}^{N} \gamma\left(\Delta, \delta_{i}, 1-\sigma_{i}\right) P\left(\boldsymbol{\sigma}_{i}\right)$.

Knowing $U_{i j}$, Eq. (28) can be solved with standard Monte-Carlo sampling techniques, allowing us to treat systems up to several $10^{5}$ atoms.

We emphasize that the description presented above is not restricted to the three-level scheme considered in this work. It can, e.g., also be applied for a direct excitation of the Rydberg state from the ground state (two-level scheme) provided that the atomic coherences are damped out fast enough to not significantly affect the population dynamics of the Rydberg state (e.g., if the bandwidth of the excitation laser is larger than the Rabi frequency of the transition). For a single-step excitation scheme the (de)-excitation rates are given by

$$
\gamma_{\uparrow}=\gamma_{\downarrow}=\frac{2 \Gamma \Omega^{2}}{\Gamma^{2}+4 \Delta^{2}} ;
$$

where $\Gamma$ is the measured width of the excitation line.

\section{Determination of the Rydberg-Rydberg interaction}

An accurate determination of the interaction potential $U_{i j}$ is challenging due to the mixing of a large number of electronically excited molecular potential curves. Results from a perturbative treatment exist for the $r_{i j} \rightarrow \infty$ asymptote of the alkali-metal atoms [16] and for the level shifts of $\mathrm{Rb}$ [17] as well as calculations for Cs based on the diagonalization of the interaction Hamiltonian of two highly excited atoms using a large number $(\sim 5000)$ of pair states as basis [18]. In the latter spirit, a simple picture was formulated in [19] for $\mathrm{Rb}$ that allows for an intuitive understanding of the basic dependence of $U_{i j}$ on $r_{i j}$ and on the principal quantum number $n$ of the Rydberg state.

Following [19], a pair of Rydberg atoms in states $|a\rangle$ and $|b\rangle$ at distance $r_{i j}$ experiences a shift $U_{i j}$ of its electronic energy due to an induced dipole coupling $V_{i j}=$ $\mu_{a a^{\prime}} \mu_{b b^{\prime}} / r_{i j}^{3}$ to an energetically close pair of states $\left|a^{\prime}\right\rangle$ and $\left|b^{\prime}\right\rangle$. The shift is given by the eigenvalues

$$
U_{i j}=\frac{1}{2}\left(\delta_{0} \pm \sqrt{\delta_{0}^{2}+4 V_{i j}^{2}}\right)
$$

of the two-state Hamiltonian matrix

$$
\mathcal{H}=\left(\begin{array}{cc}
0 & V_{i j} \\
V_{i j} & \delta_{0}
\end{array}\right),
$$

where $\delta_{0}$ is the asymptotic $\left(r_{i j} \rightarrow \infty\right)$ difference between the energies of the two pairs.

For a pair $|n s, n s\rangle$ of two atoms in the $n s$ state, the relevant dipole coupling is to the energetically close pair $\left|(n-1) p_{3 / 2}, n p_{3 / 2}\right\rangle$. For an arbitrary but fixed quantum number $n_{0}$ we may define $\mu^{2}\left(n_{0}\right) \equiv \mu_{n_{0} s\left(n_{0}-1\right) p} \mu_{n_{0} s n_{0} p}$. The interaction strength for other Rydberg levels $n$ then follows from the scaling [1]

$$
\begin{aligned}
& \mu^{2}(n)=\mu^{2}\left(n_{0}\right)\left(\frac{n^{*}}{n_{0}^{*}}\right)^{4} \\
& \delta_{0}(n)=\delta_{0}\left(n_{0}\right)\left(\frac{n_{0}^{*}}{n^{*}}\right)^{3},
\end{aligned}
$$

where $n^{*}=n-\eta$ includes the appropriate quantum defect $\eta$ (for the $n s$ states of Rb $\eta=3.13$ ). For $r_{i j} \rightarrow \infty$ one recovers the familiar van der Waals $r^{-6}$-dependence and the dominant $n^{11}$ scaling for the pair interaction $U_{i j}$. For Rb we will use in the following the values $\mu^{2}\left(n_{0}\right)=843800$ a.u. and $\delta_{0}\left(n_{0}\right)=-0.0378$ a.u. for $n_{0}=48$ from [19].

\section{AN ACCURATE TREATMENT OF TWO INTERACTING ATOMS}

As a test for our rate equation approach in the case of interacting atoms, we have numerically solved the full quantum master equation (22) and the rate equation (28) for two interacting atoms separated by an interatomic distance $r$. The quantity directly accessible in the experiments is the fraction of excited atoms $f_{e}$. It is shown in Fig. $3 \mathrm{k}$ and Fig. $3 \mathrm{~b}$ as a function of the principal quantum 

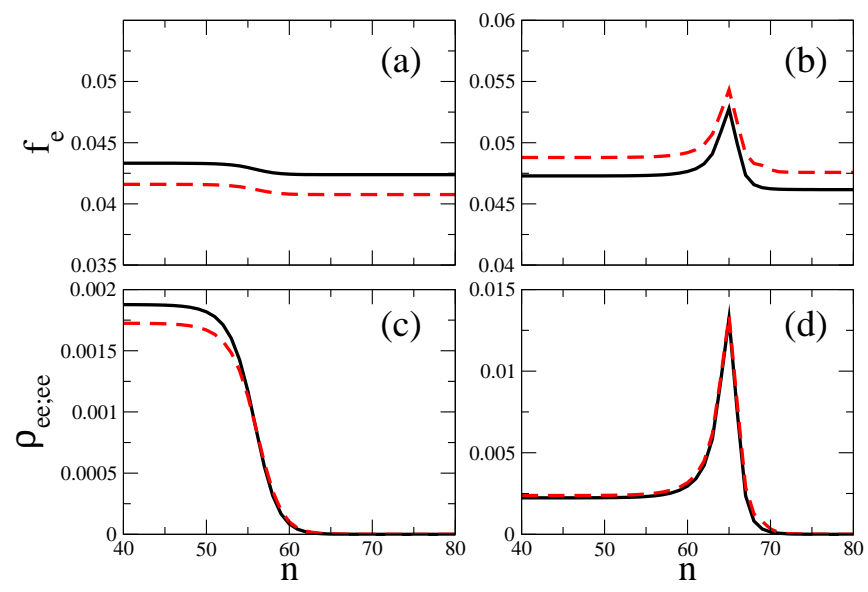

FIG. 3: Comparison of the solutions of the master equation (22) (dashed lines) and the rate equation (28) (solid lines) for two interacting atoms at distance $r=5 \mu \mathrm{m}$. Upper graphs $(\mathrm{a}, \mathrm{b})$ show the fraction of excited atoms $f_{e}$, lower graphs $(\mathrm{c}, \mathrm{d})$ the probability $\rho_{e e ; e e}$ that both atoms are in the Rydberg state as function of the principal quantum number $n$ for a pulse length $\tau=2 \mu \mathrm{s}$. The parameters of $(\mathrm{a}, \mathrm{c})$ and $(\mathrm{b}, \mathrm{d})$ are those of Figs. $2 \mathrm{a}$ and $2 \mathrm{~b}$, respectively.

number $n$ for excitation parameters used in the experiments [10] and [8], respectively. The overall agreement between the exact result and our approximation is very good and the discrepancy of only a few percent between the solutions is comparable to that of the single-atom calculations (c.f. Fig. 2, note the different scaling of the ordinate) and practically independent of the interaction strength. This indicates that most of the deviation is a consequence of the approximations already introduced at the single-atom level.

For both parameter sets we see a suppression in $f_{e}$ for large $n$, i.e., an excitation blockade. Additionally, in the case where the single-atom excitation spectrum shows a double-peak structure [Fig. [3b], there is an excitation enhancement for a certain $n$. Its actual value depends on the separation $r$ of the atoms, so that in a gas this "antiblockade" will be smeared out due to the wide distribution of mutual atomic distances. However, for atoms regularly arranged in space, i.e., on a lattice where the interatomic distances are fixed, the antiblockade should be clearly visible [15]. To verify that the observed (anti)blockade in $f_{e}$ is really a suppression (enhancement) of Rydberg pairs we have plotted the probability $\rho_{e e ; e e}$ that both atoms are in the Rydberg state. Indeed, we observe a complete suppression of the pair state in the blockade regime (Fig. 35) and the antiblockade peak (Fig. 3 d) as well as a good agreement between the solutions of the master and the rate equation in both cases.

Neglecting two-photon transitions (the second term in Eq.(24)) is the central approximation which we make in the description of the dynamics of the interacting system. In fact, these processes can be dominant, if the two-photon detuning vanishes far away from resonance, i.e., if $\Delta_{2 p h} \equiv 2 \Delta+U(r)=0$ for $|\Delta| \gg 0,|U(r)| \gg 0$. This is clearly seen in Fig. 彺, where $\rho_{e e ; e e}$ is shown as a function of the laser detuning $\Delta$ for two atoms separated by $r=5 \mu \mathrm{m}$. The solution of the master equation exhibits a triple peak structure with the central peak located at $\Delta=-U(r) / 2$ (c.f. Eq.(24)), which is not present in the solution of the rate equation. However, the probability for this two-photon transition is too small to be visible in the signal of the total probability $f_{e}$ that the atoms are in the Rydberg state (see inset).

Increasing the interatomic distance to $r=7 \mu \mathrm{m}$, i.e., decreasing the interaction strength, we expect that the blockade mechanism becomes ineffective and the contribution of Rydberg pairs to $f_{e}$ becomes relevant. This is indeed reflected in the fact that the peak of $\rho_{e e ; e e}$ in Fig. $4 \mathrm{~b}$ is orders of magnitude higher than in Fig. 4a. Here, however, the atoms are successively excited to the Rydberg state by two single-photon transitions. Hence, the peak in $\rho_{e e ; e e}$ is correctly reproduced by the rate equation.

\section{RYDBERG EXCITATION IN LARGE ENSEMBLES AND COMPARISON WITH THE EXPERIMENT}

\section{A. Dipole blockade}

\section{The density of Rydberg atoms}

We have calculated the density of Rydberg atoms as a function of the peak density of a Rb gas in a MOT according to Eq.(28) for excitations to the $62 S$ and $82 S$ state via the two-step excitation scheme as measured in [8].

More specifically, we have determined the Rabi frequency $\Omega$ of the first excitation step by using the data for the $5 S_{1 / 2}(F=2) \rightarrow 5 P_{3 / 2}(F=3)$ trapping transition of ${ }^{87} \mathrm{Rb}[20]$ and by taking the intensity of the MOT lasers from the experiment [21]. The measurment of $\Omega$ as a function of the intensity of the MOT lasers using the Autler-Townes splitting of a Rydberg line [21] is in very good agreement with our result.

To obtain the coupling strength $\omega$ of the Rydberg transition we have fitted it to the low-intensity measurements in [8] using our rate equation and scaled the result to high intensities and/or excitations to different principal quantum numbers.

Fig. 5 shows the results of our calculations and the experiment. Although we see a qualitative agreement we predict Rydberg densities about twice as large as the measured ones. As the curves for both principal quantum numbers exhibit the same deviation from the measured data, it is tempting to scale our results to the experimental points using a common factor. Note, however, that without other influences in the experiment, there is no free parameter in our description that would justify such a scaling. 

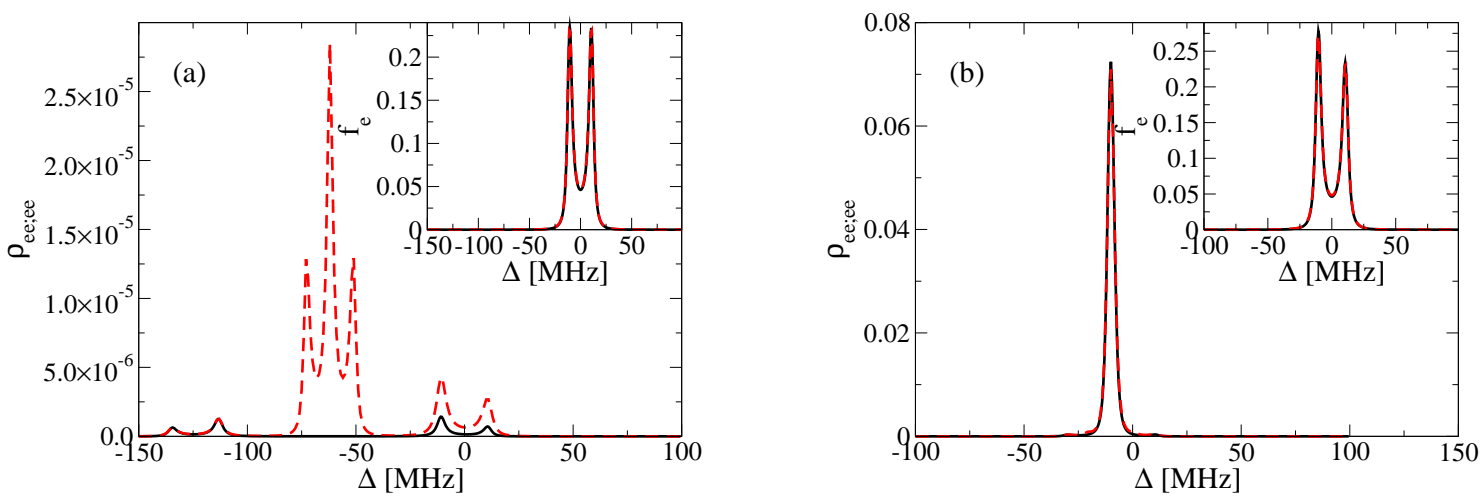

FIG. 4: Probability $\rho_{e e ; e e}$ that both atoms are in the Rydberg state $82 S$ as a function of the laser detuning $\Delta$ after an excitation time of $\tau=2 \mu \mathrm{s}$ at an interatomic distance of $r=5 \mu \mathrm{m}$ (a) and $r=7 \mu \mathrm{m}$ (b). Solid lines are the solutions of Eq.(28), the dashed lines of Eq.(22). The excitation parameters are those of Fig. 2 $\mathrm{b}$. The insets show the corresponding fraction of excited atoms $f_{e}$.

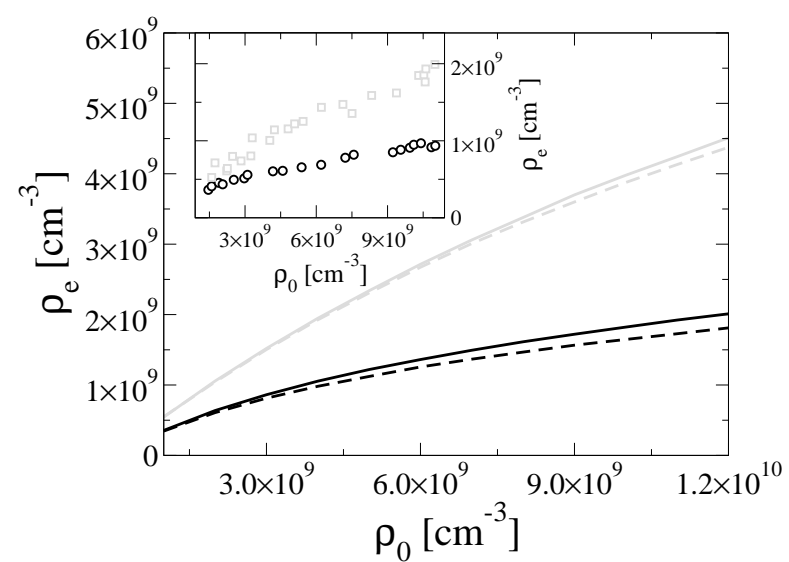

FIG. 5: Density of Rydberg atoms as a function of the peak density in the MOT for a pulse length of $\tau=20 \mu$ s for the $82 \mathrm{~S}$ (black) and the 62S state (gray) of Rb. Circles: experimental data taken from [8]. Lines: Calculations using different models for the pair interactions potential: two-state model of ref. [19] (solid) and pure van der Waals interaction from perturbative treatment [16] (dashed).

In the following we estimate the quantitative influence which several effects could have on the results presented.

\section{The influence of different Rydberg-Rydberg interactions}

The "exact" Rydberg-Rydberg interaction may differ from the one we have used in our description. To assess the impact of such a difference, we have performed our calculations with the simple two-state model discussed above (solid lines in Fig. 5) and assuming a "pure" van der Waals interaction, $-C_{6} / r^{6}$, between the Rydberg atoms (dashed lines in Fig. 5). The interaction coeffi- cients $C_{6}(n)$ for the latter are calculated in second-order perturbation theory for $r \rightarrow \infty$ and have been taken from [16]. The interaction strength for the $n S$ states calculated in this way is considerably larger than the one from the two-state model (e.g., for the $82 S$ state the difference in $U(r)$ at $r=10 \mu \mathrm{m}$ is roughly a factor of 2.5 and increases with decreasing $r$ ). Yet, the final results for the Rydberg population differ only slightly (see Fig. (5). We conclude that $\rho_{\mathrm{e}}$ is relatively robust against changes in the interaction strength. This is due to the fact that the measurement of the Rydberg density as a function of the ground state density does not probe the exact shape of the interaction potential but rather the critical distance $r_{\mathrm{c}}$ at which the energetic shift caused by the interaction becomes larger than half the width of the spectral line $(\approx 20 \mathrm{MHz})$. For $U(r)$ determined in perturbation theory and estimated by the two-state approximation $r_{\mathrm{c}} \approx 8 \mu \mathrm{m}$ and $r_{\mathrm{c}}=7 \mu \mathrm{m}$, respectively, for the $82 \mathrm{~S}$ state, so that significant differences emerge only for large densities.

\section{The influence of ions}

Another effect, so far not accounted for, is the presence of ions. The excitation pulse length used in [8] was $20 \mu \mathrm{s}$. For pulse durations that long, it was shown that a significant amount of Rydberg atoms can undergo ionizing collisions even for a repulsive Rydberg-Rydberg interaction [19, 22]. The presence of ions in the system influences the excitation dynamics due to the polarizing effect of the electric field of the ions on the highly susceptible Rydberg atoms. The Rydberg-ion interaction $\left(\propto r^{-4}\right)$, therefore, leads to an additional energetic shift of the Rydberg levels and, thus, can lead to an enhanced excitation suppression.

To see if the presence of ions can account for the difference between our results and the measured data, we 


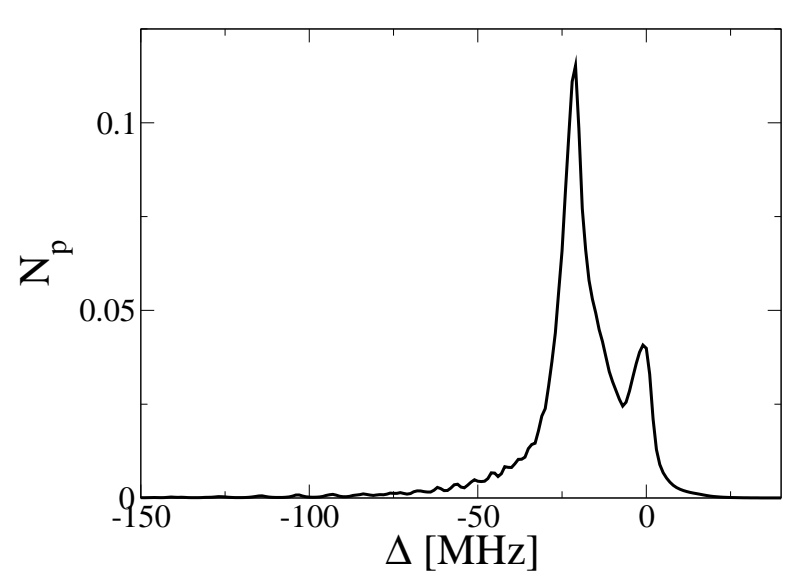

FIG. 6: Estimated average number $N_{p}$ of $n=82$ Rydberg pairs excited by multi-photon transitions as a function of the laser detuning $\Delta$ after $\tau=20 \mu$ s for a ground state peak density $\rho_{0}=10^{10} \mathrm{~cm}^{-3}$.

have performed calculations in which we have replaced up to $20 \%$ of the Rydberg atoms by ions. The change in the results compared to the situation without ions is comparable to that of stronger Rydberg-Rydberg interaction discussed above. Therefore, ions can be ruled out as a source for the discrepancy between our and the experimental results.

\section{The influence of multiphoton transitions}

The excitation line profiles presented in [8] showed an enormous broadening for measurements at high densities. In contrast, the line profiles that we have calculated with the present approach are much narrower, in accordance with the simulations reported in ref. [13].

The strong line broadening in the experiment could be due to non-resonant effects, such as multiphoton transitions, not included in our rate description (see discussion in section IIII). To estimate their possible influence, we have to determine first the number of Rydberg pairs which could be excited by these transitions. To this end, we have determined the number of (ground state) atoms $n_{p}(r) \Delta r$ which form a pair with a distance between $r$ and $r+\Delta r$ in the excitation volume, from the pair density $n_{p}(r)$. Furthermore, we have calculated the probability $\rho_{e e ; e e}$ for a pair of atoms to be in the Rydberg state after $\tau=20 \mu$ s by solving the quantum master equation $\left(w_{p}^{\mathrm{ME}}\right)$ and the rate equation $\left(w_{p}^{\mathrm{RE}}\right)$ for two atoms as a function of the laser detuning $\Delta$ and interatomic distance $r$ (c.f. Fig. 4). The difference $w_{p}(r, \Delta)=w_{p}^{\mathrm{ME}}(r, \Delta)-w_{p}^{\mathrm{RE}}(r, \Delta)$ should give a rough estimate for the probability of a Rydberg pair being excited by a multi-photon transition. The average number of such pairs as a function of $\Delta$ can then be estimated by $N_{p}(\Delta)=\sum_{i} w_{p}\left(r_{i}, \Delta\right) n_{p}\left(r_{i}\right) \Delta r$.

Fig. 6] shows that for a sample with ground state peak density $\rho_{0}=10^{10} \mathrm{~cm}^{-3}$ our estimate yields a negligible number of Rydberg pairs excited by multi-photon transitions after $20 \mu \mathrm{s}$. Although these estimates are rather crude, the result shows that multi-photon effects are too small to explain the broadening of the excitation line profile in the experiment [8].

In summary, the unexplained line broadening and the difference between experiment and theory in the Rydberg populations make it likely that some additional, presently not known process, has contributed significantly to the results obtained in [8].

\section{B. Antiblockade}

\section{Lattice configurations}

The discussion in Sec. [II has shown that the structure of the single-atom excitation line strongly influences the excitation dynamics in the interacting system. Even on resonance, the Rydberg-Rydberg interaction can cause an excitation enhancement, if the spectral line exhibits a double peak structure. This antiblockade occurs whenever the interaction-induced energetic shift $\Delta_{i}$ for an atom at position $\mathbf{r}_{i}$ matches the detuning $\Delta_{\max }$ at which the single-atom excitation probability has its maximum value.

In the gas phase, where the mutual atomic distances are broadly distributed, the antiblockade can hardly be observed by measuring the fraction of excited atoms $f_{e}$, as the condition $\Delta_{i}=\Delta_{\max }$ is only met by relatively few atoms [15]. In contrast, if the atoms are regularly arranged in space, e.g., with the help of an optical lattice produced by $\mathrm{CO}_{2}$ lasers 23], one should clearly observe peaks in $f_{e}$ for certain $n$ (see Fig. $7 \mathrm{~b}$ ). The peak positions can easily be determined by analyzing the geometry of the underlying lattice. Moreover, the effect is quite robust against lattice defects (unoccupied lattice sites) and should therefore be experimentally realizable. A more detailed discussion can be found in [15].

The underlying lattice structure allows for a statistical interpretation of the antiblockade as "clustering" of Rydberg atoms. Using the terminology of percolation theory, we define a cluster of size $s$ as group of $s$ nearest neighbor sites occupied by Rydberg atoms. For negligible Rydberg-Rydberg interaction the excitation of atoms on a lattice is analogous to the situation encountered in classical (site-)percolation theory. This is seen in Fig. [7b, where a histogram of the average number $n_{s}$ of $s$-clusters per lattice site as function of the cluster size (normalized to the number of 1-clusters, i.e., isolated Rydberg atoms) is shown for atoms excited to the state $n=40$. The shaded area represents the prediction of percolation theory 24] for the same number of isolated Rydberg atoms per site and shows good agreement with the "measured" data. In the antiblockade regime $(n=65$, Fig. $7 \mathrm{k})$ we observe a broadening of the cluster size distribution and a significant enhancement of larger Rydberg clusters, while in the blockade regime $(n=68$, Fig. $7 \mathrm{~d})$ a quenching of 

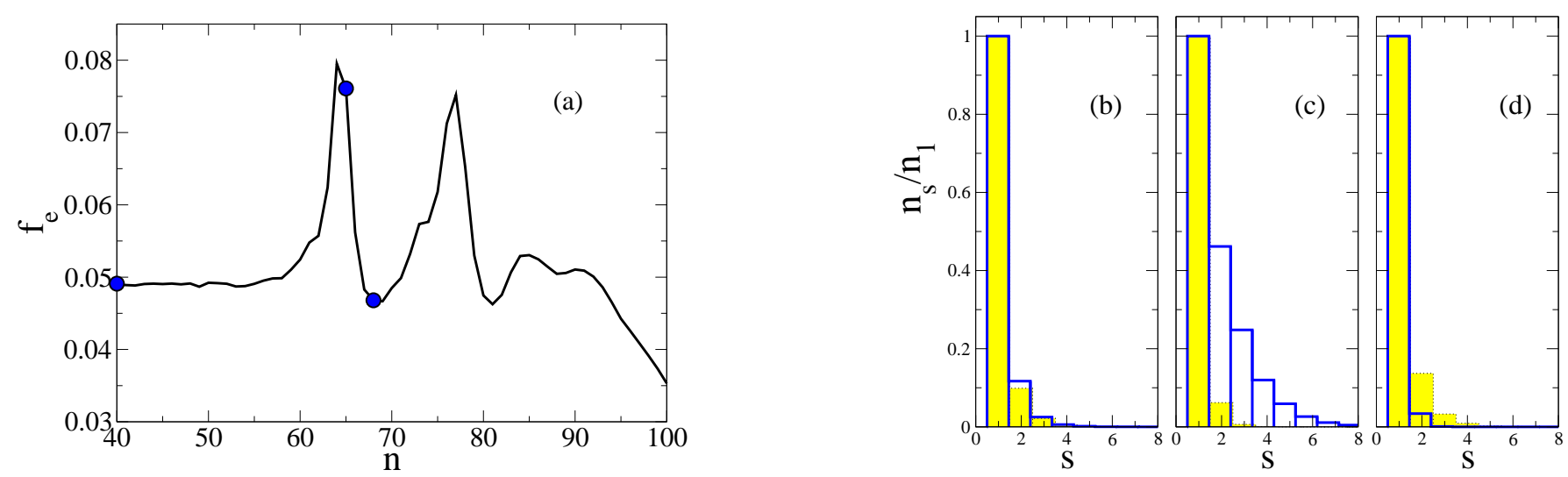

FIG. 7: (a) Fraction of excited atoms for atoms on a simple cubic lattice with $20 \%$ unoccupied sites as function of the principal quantum number $n$. The lattice constant is $a=5 \mu \mathrm{m}$, all other parameters are those of Fig. 20 b. (b-d) Corresponding number of "Rydberg clusters" per lattice site $n_{s}$ normalized to the number of 1-clusters (i.e. isolated Rydberg atoms) $n_{1}$ as a function of the cluster size $s$ for principal quantum number $n=40$ (b), $n=65$ (c) and $n=68$ (d). The shaded areas represent predictions from percolation theory [24] for a system with the same number of isolated Rydberg atoms (1-clusters) per lattice site.

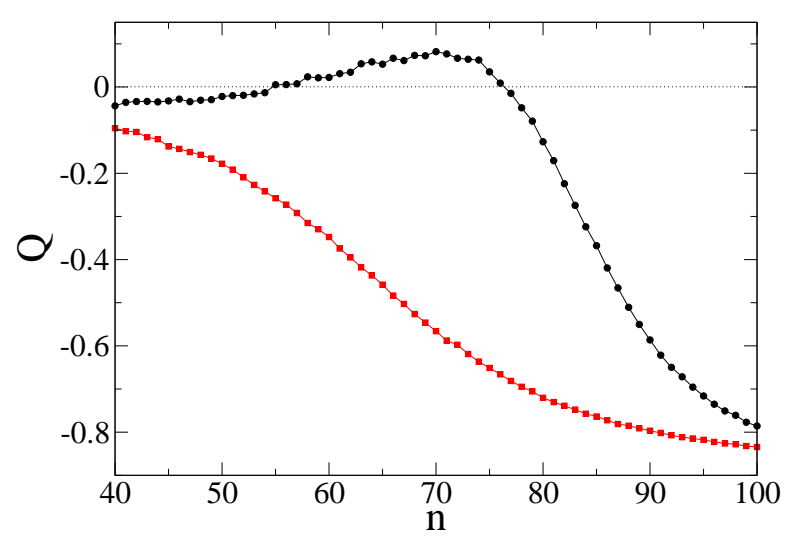

FIG. 8: Comparison of the $Q$-parameter in the blockade (squares) and antiblockade (circles) configuration as a function of the principal quantum number $n$ for a sample with a homogeneous atomic density $\rho_{0}=8 \times 10^{9} \mathrm{~cm}^{-3}$ and for an excitation pulse length $\tau=2 \mu \mathrm{s}$. $Q$ was determined by $10^{5}$ successive measurements of $\left\langle N_{r y d}\right\rangle$ and $\left\langle N_{r y d}^{2}\right\rangle$. The Rabi frequencies $(\Omega, \omega)$ are: $(4.0,0.24) \mathrm{MHz}$ (squares) and $(22.1,0.8)$ $\mathrm{MHz}$ (circles).

the distribution and an enhancement of the probability to excite isolated Rydberg atoms is evident.

\section{Random gases}

Based on the solution of a many-body rate equation using Monte Carlo sampling, the present approach is particularly well suited to determine statistical properties of interacting Rydberg gases.

In [10] the distribution of the number of Rydberg atoms was measured as function of the interaction strength. The distributions obtained were quantified by
Mandel's $Q$-parameter

$$
Q=\frac{\left\langle N_{e}^{2}\right\rangle-\left\langle N_{e}\right\rangle^{2}}{\left\langle N_{e}\right\rangle}-1
$$

where $N_{e}$ is the number of Rydberg atoms and $\langle\ldots\rangle$ denotes the average over the probability distribution. The $Q$-parameter measures the deviation of a probability distribution from a Poissonian, for which it is zero, whereas for a super-(sub-)Poissonian it is positive (negative). The experiment showed a quenching of the Rydberg number distribution, i.e., a decrease of $Q$, for increasing interaction strength as theoretically confirmed [11, 12]. The differences between the theoretical calculations $(Q<0$, for all $n)$ and the measured values $(Q>0)$ can be attributed to shot-to-shot fluctuations of the number of ground state atoms in the experiment [25].

The excitation parameters in [10] were in the blockade regime, where the single-atom excitation line exhibits a single peak at $\Delta=0$. Therefore, there is a volume ("correlation hole") around each Rydberg atom, where the excitation of additional atoms is strongly suppressed. On the other hand, in the parameter regime of the antiblockade, where the excitation line shows a double peak structure, there is in addition a shell around each Rydberg atom, in which additional excitations are strongly enhanced. Thus, the statistics of the Rydberg excitations should depend on the structure of the single-atom excitation line and the antiblockade can be detected indirectly even in the gas phase by measuring the atom counting statistics.

Figure 8 shows the calculated $Q$-parameter as a function of the principal quantum number $n$ for the blockade and antiblockade regime. In the blockade configuration (squares) one observes a monotonic decrease of $Q$ with $n$ in accordance with the measurements in [10]. In the antiblockade regime (circles), however, $Q$ is nonmonotonic, i.e., the distribution is slightly broadened, 
and the quenching starts at much higher $n$. Although the broadening of the distribution may be difficult to observe experimentally, the difference in the functional form of $Q(n)$ provides a clear experimental signature in a mesoscopic region of the MOT, where the atomic density is approximately homogeneous.

\section{CONCLUSIONS}

We have developed a simple approach, which allows one to describe the dynamics in ultracold gases, in which Rydberg atoms are excited via a resonant two-step transition. Starting from a quantum master equation, which incorporates the full dynamics of an interacting gas of three-level atoms, we have derived a many-body rate equation. It covers the correlated dynamics of the system, yet, it can easily be solved by Monte Carlo sampling for a realistically large number of atoms.

Our approach, valid under well defined conditions typical for experiments, is based upon two approximations: (i) an adiadabtic approximation on the single-atom level to eliminate the atomic coherences and (ii) the negligence of multi-photon transitions in the interacting system. Solving the problem of two interacting atoms exactly with a quantum master equation we could show that the approximate solution based on the rate equation is in very good agreement with the exact result.

The present approach is capable of reproducing the partial excitation blockade observed in 8] qualitatively.

Qualitatively in accordance with our calculations regarding the excitation line shape and the so called Qparameter are also the experimental results of [10].

Finally, the careful analysis of the two-step excitation scheme has lead to the prediction of an antiblockade effect due to an Autler-Townes splitting of the intermediate level probed by the Rydberg transition in the appropriate parameter regime. This antiblockade should be directly observable for a lattice gas, realized, e.g., with an optical lattice. As we have demonstrated, it could also be observed indirectly in the gas phase through the atom counting statistics which differs qualitatively from its counterpart in the blockade regime.

\section{APPENDIX: EXPRESSIONS FOR $\rho_{\text {ee }}^{\infty}$ AND $\gamma_{\uparrow}$}

The steady state solution of the OBE (6) for the Rydberg population is

$$
\rho_{\mathrm{ee}}^{\infty}=\frac{\Omega^{2}\left(\Omega^{2}+\omega^{2}\right)}{\left(\Omega^{2}+\omega^{2}\right)^{2}+4 \Delta^{2}\left(\Gamma^{2}+2 \Omega^{2}\right)} .
$$

The excitation rate in (11) can be written as

where

$$
\gamma_{\uparrow}=\frac{2 \Gamma(\omega \Omega)^{2}\left(\Omega^{2}+\omega^{2}\right)}{a_{0}+a_{2} \Delta^{2}+a_{4} \Delta^{4}},
$$

$$
\begin{aligned}
a_{0}= & \left(\Omega^{2}+\omega^{2}\right) \\
& \cdot\left[\left(\omega^{2}-2 \Omega^{2}\right)^{2}+2 \Gamma^{2}\left(\Omega^{2}+\omega^{2}\right)\right] \\
a_{2}= & 8\left(\Gamma^{4}-4 \Omega^{4}\right) \\
& +4 \omega^{2}\left(\Gamma^{2}+4 \Omega^{2}\right)+8 \omega^{4} \\
a_{4}= & 32\left(\Gamma^{2}+2 \Omega^{2}\right) .
\end{aligned}
$$

[1] T. F. Gallagher, Rydberg atoms (Cambridge University Press, 1994).

[2] W. R. Anderson, J. R. Veale, and T. F. Gallagher, Phys. Rev. Lett. 80, 249 (1998).

[3] I. Mourachko, D. Comparat, F. de Tomasi, A. Fioretti, P. Nosbaum, V. M. Akulin, and P. Pillet, Phys. Rev. Lett. 80, 253 (1998).

[4] M. D. Lukin, M. Fleischhauer, R. Côté, L. M. Duan, D. Jaksch, J. I. Cirac, and P. Zoller, Phys. Rev. Lett. 87, 037901 (2001).

[5] I. Bouchoule and K. Mølmer, Phys. Rev. A 65, 041803 (2002).

[6] M. Saffman and T. G. Walker, Phys. Rev. A 66, 065403 (2002).

[7] D. Tong, S. M. Farooqi, J. Stanojevic, S. Krishnan, Y. P. Zhang, R. Côté, E. E. Eyler, and P. L. Gould, Phys. Rev. Lett. 93, 063001 (2004).

[8] K. Singer, M. Reetz-Lamour, T. Amthor, L. G. Marcassa, and M. Weidemüller, Phys. Rev. Lett. 93, 163001 (2004).

[9] T. Vogt, M. Viteau, J. Zhao, A. Chotia, D. Comparat, and P. Pillet, Phys. Rev. Lett. 97, 083003 (2006).

[10] T. Cubel-Liebisch, A. Reinhard, P. R. Berman, and
G. Raithel, Phys. Rev. Lett. 95, 253002 (2005).

[11] C. Ates, T. Pohl, T. Pattard, and J. M. Rost, J. Phys. B 39, L233 (2006).

[12] J. V. Hernández and F. Robicheaux, J. Phys. B 39, 4883 (2006).

[13] F. Robicheaux and J. V. Hernández, Phys. Rev. A 72, 063403 (2005).

[14] C. Cohen-Tannoudji, J. Dupont-Roc, and G. Grynberg, Atom-photon interactions: basic processes and applications (John Wiley \& Sons, 1992).

[15] C. Ates, T. Pohl, T. Pattard, and J. M. Rost, Phys. Rev. Lett. 98, 023002 (2007).

[16] K. Singer, J. Stanojevic, M. Weidemüller, and R. Côté, J. Phys. B 38, S295 (2005).

[17] A. Reinhard, T. C. Liebisch, B. Knuffman, and G. Raithel, Phys. Rev. A 75, 032712 (2007).

[18] A. Schwettmann, J. Crawford, K. R. Overstreet, and J. P. Shaffer, Phys. Rev. A 74, 020701 (2006).

[19] W. Li, P. J. Tanner, and T. F. Gallagher, Phys. Rev. Lett. 94, 173001 (2005).

[20] D. A. Steck, Rubidium 87 D line data (2003), URL http://steck.us/alkalidata. 
[21] M. Weidemüller, private communication.

[22] T. Amthor, M. Reetz-Lamour, S. Westermann, J. Denskat, and M. Weidemuller, Phys. Rev. Lett. 98, 023004 (2007).

[23] S. Friebel, C. D'Andrea, J. Walz, M. Weitz, and T. W. Hänsch, Phys. Rev. A 57, R20 (1998).
[24] M. F. Sykes, D. S. Gaunt, and M. Glen, J. Phys. A 9, 1705 (1976).

[25] T. Cubel-Liebisch, A. Reinhard, P. R. Berman, and G. Raithel, Phys. Rev. Lett. 98, 109903 (2007). 